Universidad de Lima

Facultad de Psicología

Carrera de Psicología

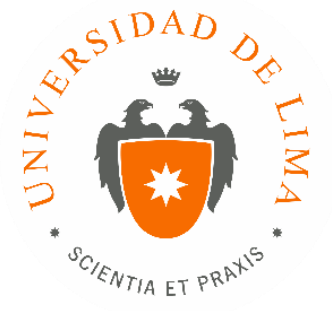

\title{
DIAGNÓSTICO DEL CLIMA ORGANIZACIONAL
}

Trabajo de suficiencia profesional para optar el título profesional de Licenciado en Psicología

\section{Sandra Margoth Simón Fernández}

\section{Código 20062858}

$$
\text { Lima - Perú }
$$

Febrero de 2019 



\section{DIAGNÓSTICO DEL CLIMA ORGANIZACIONAL}




\section{TABLA DE CONTENIDO}

INTRODUCCIÓN

CAPÍTULO I: IDENTIFICACIÓN DEL PROBLEMA .................................................. 8

CAPÍTULO II: DESCRIPCIÓN DE LAS ACTIVIDADES Y TAREAS REALIZADAS ..... 11

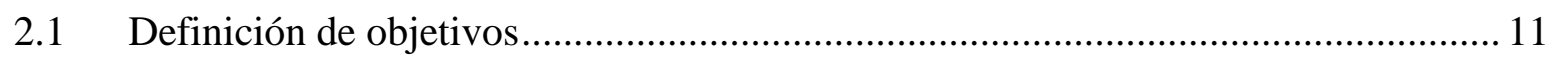

2.2 Elección del método de recogida de datos.......................................................... 12

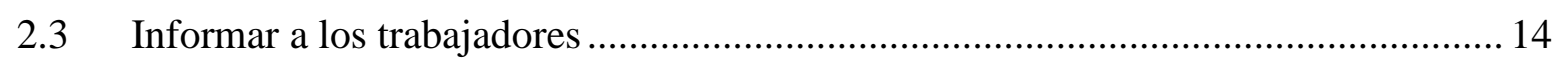

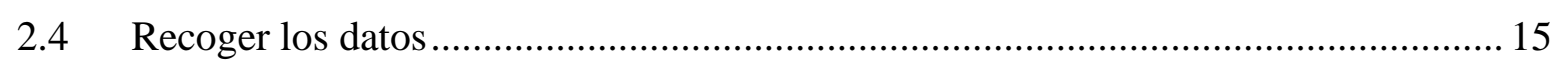

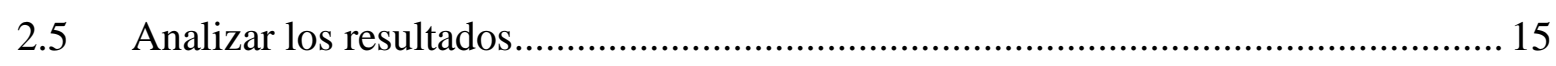

2.6 Entregar los resultados a líderes y comunicar a toda la organización ....................... 16

CAPÍTULO III: RESULTADOS DE LA INTERVENCIÓN ............................................ 18

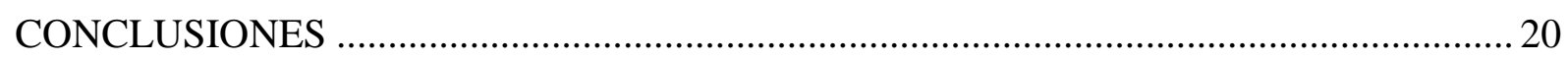

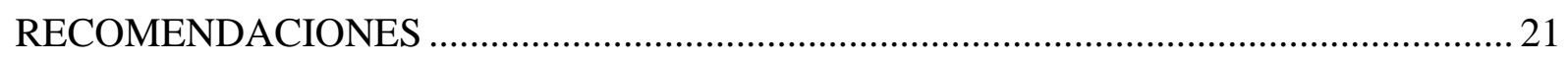

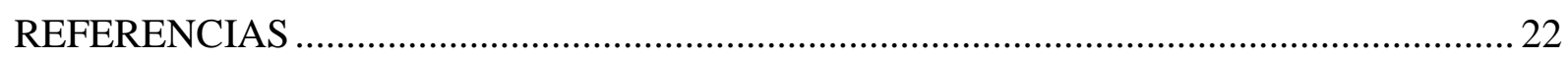

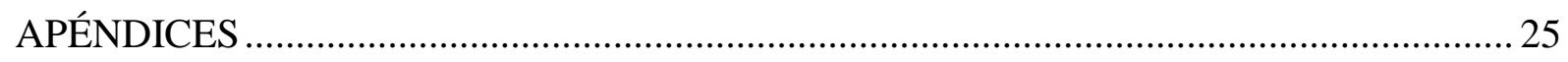




\section{ÍNDICE DE FIGURAS}

Figura 1. Modelo de efectividad organizacional ........................................................... 12

Figura 2. Matriz soporte para el éxito vs. compromiso ..................................................... 13 


\section{ÍNDICE DE APÉNDICES}

APÉNDICE 1: CARTA DE AUTORIZACIÓN DE LA EMPRESA ...................................... 26

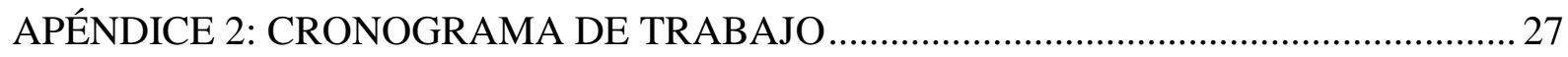

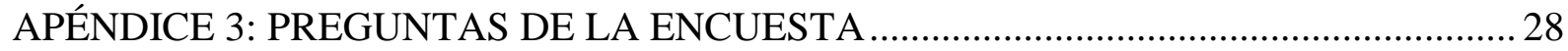

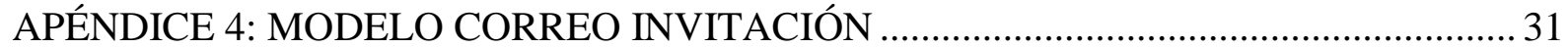

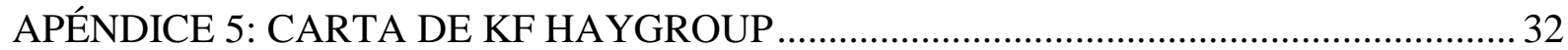




\section{INTRODUCCIÓN}

Todas las organizaciones desean cumplir con sus objetivos a largo plazo. Por ello, necesitan personas comprometidas y que cuenten con las competencias adecuadas para alcanzar sus metas. Para ello, el clima laboral de la organización debe ser adecuado y, con el fin de mejorarlo, se realizan diagnósticos de clima laboral que permiten conocer las percepciones de los trabajadores y así poder realizar planes de acción a la medida. Estos deben generar bienestar y estar alineados a las exigencias del mundo actualmente: globalización, tecnología y ecología (D’Alessio, 2017). Los trabajadores, por su parte, presentan necesidades individuales, metas y expectativas distintas, y las formas de lograr la satisfacción y motivación de cada uno son, por tanto, diversas. Como resultado, las organizaciones se esfuerzan por comprender las necesidades, intereses y deseos de las personas, ofreciendo a cada trabajador el mejor reconocimiento de acuerdo con sus expectativas (Pereda, Berrocal y Alonso, 2014; Peña Estrada, 2015). Los conceptos de motivación y satisfacción laboral son distintos, pero se encuentran relacionados. Para Robbins y Judge (2013), la motivación es el proceso que determina la intensidad, dirección y persistencia del esfuerzo de una persona hacia el logro de una meta, mientras que la satisfacción laboral es una actitud, un sentimiento positivo respecto del trabajo, que resulta de la evaluación de las características de este. Como indican Pereda et al. (2014), la motivación hace referencia al impulso, mientras que la satisfacción es un estado que se obtiene de la experiencia laboral. Así, la búsqueda de la satisfacción motiva la acción. El presente documento, con fines académicos, presenta un caso de diagnóstico de clima organizacional: primero, identifica el problema; luego, describe las actividades y tareas realizadas; y, finalmente, se presentan los resultados de la intervención, conclusiones y recomendaciones. 


\section{CAPÍTULO I: IDENTIFICACIÓN DEL PROBLEMA}

El diagnóstico de clima organizacional fue realizado en la administración de un xxxxx

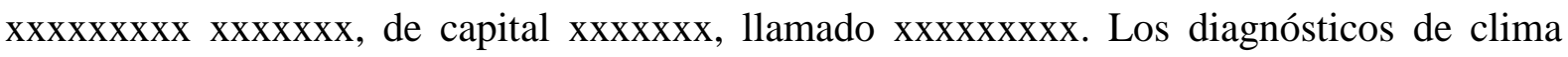
usados como referencia para la elaboración del presente documento fueron los aplicados en junio de 2017 y en mayo de 2018.

Los estudios se realizaron en un contexto político de mucha inestabilidad como consecuencia del caso Odebrecht, en el que varios políticos peruanos se han visto involucrados, incluido el presidente de la República de ese momento, Pedro Pablo Kuczynski (PPK) (CNN Español, 2017). Adicionalmente, la oposición fujimorista agravó la crisis política, lo que derivó en la renuncia de PPK en marzo del 2018, en medio de acusaciones de corrupción y sobornos (BBC Mundo, 2018). El contexto económico se vio afectado por la crisis política y el crecimiento del Producto Interno Bruto (PIB) se mantuvo en un 3\%, debido a que las inversiones públicas y privadas estaban limitadas (América Economía, 2018). El contexto social, además de verse afectado por la crisis política, se vio perjudicado por el fenómeno de El Niño costero, que dejó un saldo de más de 150 personas fallecidas, más de 4000 kilómetros de carretera destruida, más de 50,000 mil áreas de cultivo, y más de 500,000 mil casas afectadas (RPP Noticias, 2017). Vale destacar que, durante esta crisis social, se manifestaron diversas muestras de solidaridad entre la población. Posteriormente, la clasificación de Perú en la Copa Mundial de Fútbol generó un sentido de orgullo sin precedentes entre los peruanos.

En cuanto a la empresa estudiada, el xxxxxxxx es el xxxxxxxxx más grande del Perú con

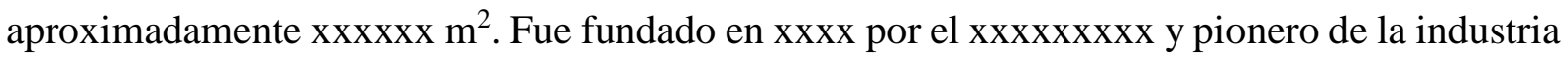

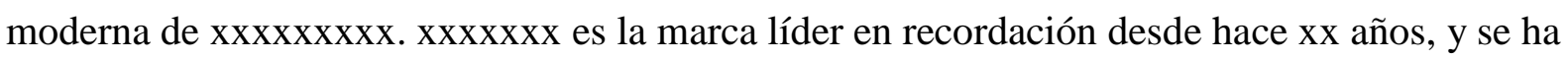
posicionado como la marca más reconocida de la industria. Así, atiende aproximadamente a xxxxx de visitantes al mes y alcanza los xxxxxx al año, con una concentración en los sectores 
socioeconómicos A, B y C1. Asimismo, ofrece el servicio de xxxxxxxxx para servicios y tiendas de las marcas más reconocidas a nivel nacional e internacional (más de xxx xxxxxx

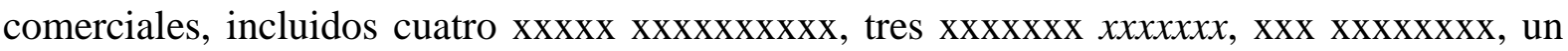
$\operatorname{xxx} x x x x)$.

Así, durante las últimas décadas, el xxxxxxxxx se ha posicionado como líder en ventas, área xxxxxxx y xxxxx de preferencia para los limeños (Equilibrium Clasificadora de Riesgo, 2018).

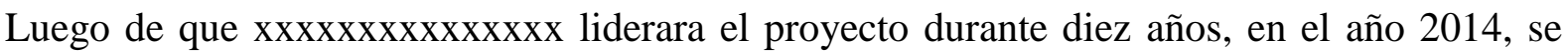
retiró y se dieron cambios estructurales importantes durante los tres años posteriores, como el cambio de dos gerentes generales, tres directores comerciales, dos CFO y varios gerentes de primera línea. Todos estos cambios fueron incentivados por el directorio. Al mismo tiempo, se

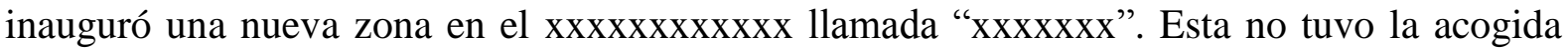
esperada, ya que no recibió el flujo esperado. Por ello, los xxxxxxxx se manifestaron insatisfechos, dado que las expectativas generadas por sus equipos comerciales no se cumplieron. Incluso, una xxxxxxxxxxxx decidió cerrar sus puertas (El Comercio, 2016). En medio de esta crisis, el equipo de Recursos Humanos decidió no aplicar diagnóstico de clima laboral en el año 2016, pues había mucha inestabilidad en la empresa, los lideres no estaban empoderados por el directorio debido a la coyuntura general, y la encuesta podría haber generado expectativas que en ese momento no se iban a poder atender.

En el año 2017, se programó aplicar la encuesta de clima laboral la primera semana de junio y, esa misma semana, el directorio decidió nombrar al que fue gerente general por 10 años, Juan

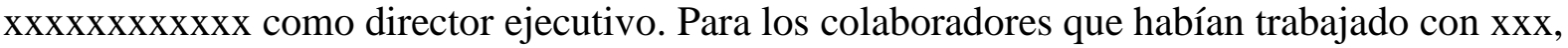
esa noticia fue recibida de manera positiva; sin embargo, entre los que no lo conocían, se generó mucha incertidumbre, pues habían entrado con la nueva gerencia general. Por ello, el nivel de satisfacción de esa encuesta fue poco favorable, y la organización cuestionó los resultados y los aspectos metodológicos de la misma. 
Los ingresos de la empresa ese año resultaron en $2.8 \%$ por encima de los ingresos del 2016, y la rentabilidad entre diciembre del 2016 y junio del 2017 se mantuvo en 58.7\%. (Equilibrium Clasificadora de Riesgo, 2018). Durante ese año, se trabajó un plan de acción sobre la base de los resultados obtenidos en la encuesta. En diciembre, el directorio nombró como gerente general nuevamente a xxx.

En el 2018, el equipo de Recursos Humanos programó aplicar la encuesta de clima laboral la primera semana de mayo. Esta debía ser adecuadamente elaborada y cumplir con las expectativas de los clientes internos de la organización. El reto de esta nueva encuesta fue que todos los líderes confiaran en la metodología, que sea comparable con la del año anterior, y que se aprobaran los resultados que arroje la misma, pues el año anterior se habían generado muchos cuestionamientos.

Se describe, a continuación, las actividades y tareas realizadas en la aplicación de la encuesta de mayo 2018, en la que se realizaron los ajustes respecto de los resultados de la anterior. 


\section{CAPÍTULO II: DESCRIPCIÓN DE LAS ACTIVIDADES Y TAREAS REALIZADAS}

\subsection{Definición de objetivos}

Todo diagnóstico de clima debe definir sus objetivos antes de comenzar el proceso. Esta etapa es fundamental, pues dichos objetivos son claves para tomar decisiones importantes y para velar por la implementación de medidas oportunas que mejoren la situación actual (Pereda et al., 2014). Particularmente, el objetivo general trazado para el diagnóstico del clima laboral 2018 del xxxxxxxxxxx fue gestionar la efectividad organizacional de los trabajadores para lograr que sea un mejor lugar para trabajar, que contribuya al mismo tiempo con el logro de los objetivos estratégicos de la empresa. Los objetivos específicos fueron los siguientes: (a) conocer el nivel de compromiso y soporte organizacional a través de una herramienta de medición confiable; (b) descubrir cómo los empleados ven la efectividad de su organización y de su ambiente de trabajo; (c) identificar cuáles son las barreras que impiden el alto desempeño de los trabajadores; (d) mejorar la retención de los empleados; (e) entender cómo mejora la efectividad de los empleados sosteniendo una mirada global de la organización; (f) identificar particularidades de cada una de las áreas y segmentos involucrados, e identificar oportunidades de mejora; (g) ayudar a los líderes a comprender cómo mejorar la efectividad de sus equipos de trabajo; (h) resaltar los elementos importantes para enfocar los recursos hacia un mayor impacto en el negocio; e (i) elaborar recomendaciones alineadas con la estrategia y desafíos de cada una de las unidades de negocio.

Las funciones realizadas por la posición fueron elaborar los objetivos del diagnóstico de clima, alineados con los objetivos de la organización, bajo supervisión de la gerencia del área en conjunto con la alta dirección. 


\subsection{Elección del método de recogida de datos}

La elección del método también es una etapa clave del proceso, ya que el mismo debe estar alineado con los objetivos del proceso y con los objetivos estratégicos de la organización. El clima puede ser estudiando de diversas maneras y, para mejorar la competitividad de la organización, es necesario conocer los factores involucrados (Govea, Domínguez y San Agustín, 2012). Los diferentes métodos para la recogida de datos son los siguientes: (a) cuestionarios, los cuales pueden ser estandarizados, como el WES, el CLA, el FOCUS-93 y el OCS, o ad hoc diseñados según los aspectos más importantes de la organización, con preguntas pueden cerradas, abiertas o mixtas; (b) entrevista individual, con la cual se obtiene información principalmente cualitativa; y (c) entrevista de grupo, que se usa como complemento del cuestionario y de la entrevista individual (Pereda et al., 2014).

En este caso, se utilizó un cuestionario ad hoc, realizado en conjunto con la consultora Korn Ferry HayGroup. Este se dividió en dos dimensiones: compromiso y soporte para el éxito. Esto permite conocer la efectividad del empleado y, de esta manera, vincularla directamente con los resultados del negocio (Korn Ferry HayGroup, 2017). En la figura 1, a continuación, se muestra el modelo de efectividad organizacional:

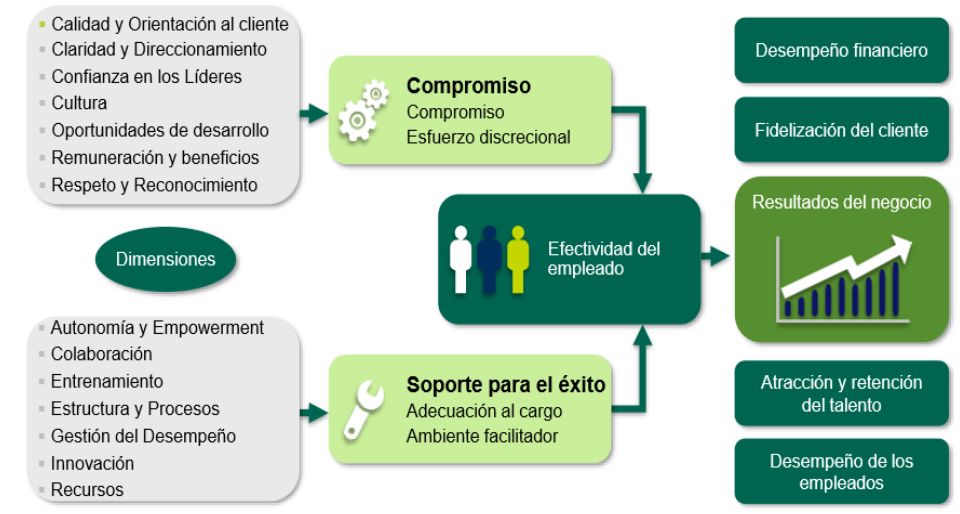

\section{\1}

Figura 1. Modelo de efectividad organizacional 
Adicionalmente, este método brinda una matriz de efectividad organizacional, la cual permite conocer la cantidad de trabajadores efectivos, indiferentes, frustrados y menos efectivos. En la figura 2, se explica a detalle la matriz en base a las dimensiones estudiadas:

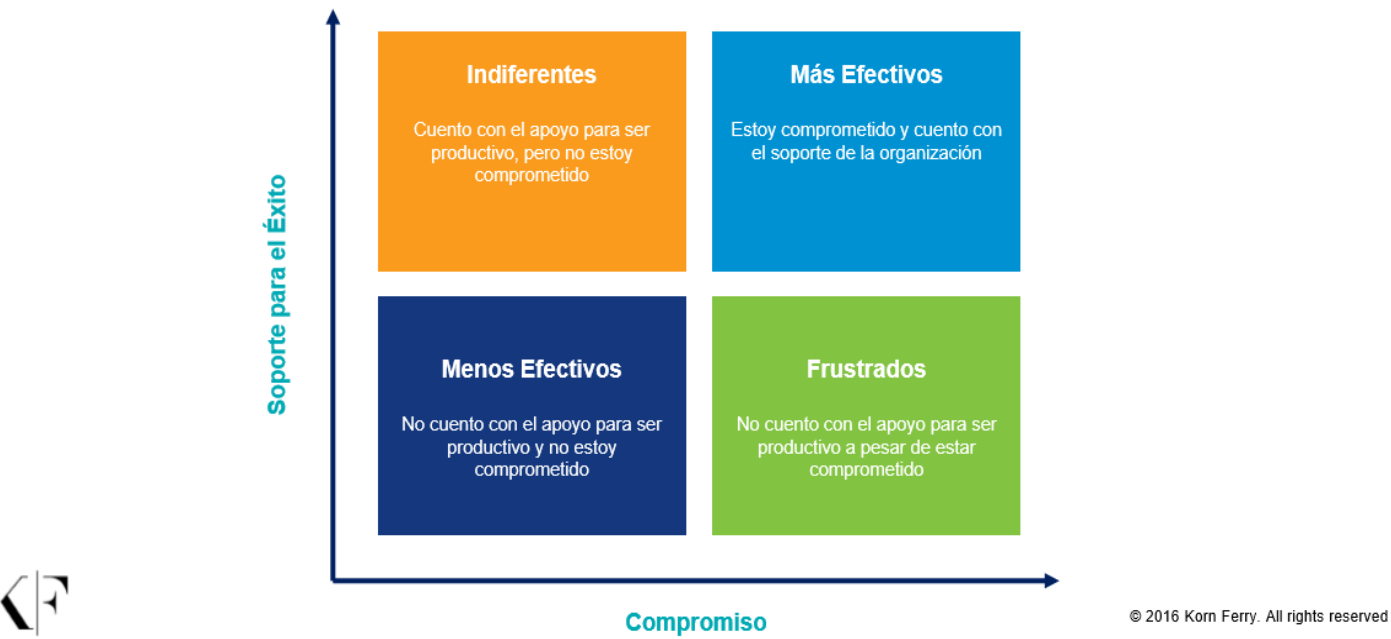

Figura 2. Matriz soporte para el éxito vs. compromiso

Las actividades realizadas en esta etapa del proceso fueron seis: a) se escogió el tipo de cuestionario ya mencionado; b) se investigó sobre los diferentes proveedores del mercado y sus metodologías, entre los que se eligió a Korn Ferry HayGroup; c) se decidió que la aplicación sería virtual; d) se definió que la escala de respuesta sería tipo Likert, con cinco categorías para expresar el grado de acuerdo o en desacuerdo; e) se elaboró un cronograma de trabajo ${ }^{1}$; f), se revisó a detalle cada pregunta del cuestionario y la segmentación que tendría la organización.

En esta última etapa, se aplicaron ajustes de la encuesta 2017 versus 2018, ya que, en el 2017, primer año que se aplicó la encuesta con Korn Ferry, las preguntas fueron revisadas por Recursos Humanos y la Gerencia General, y la segmentación fue realizada de manera personal por cada trabajador. Sin embargo, al aplicar la encuesta, se generaron muchas dudas por parte de los usuarios tanto en el momento de responder el

\footnotetext{
${ }^{1}$ Ver apéndice 2
} 
cuestionario como cuando se realizó la segmentación. Debido a ello, para la encuesta del 2018, se involucró a todos los líderes de la organización en la revisión del cuestionario y, además, se segmentó a toda la organización previamente. Para revisar el cuestionario, se analizó cada pregunta y fueron parafraseadas en caso de ser necesario; también, se agregaron nuevas preguntas para mayor entendimiento y recolección de información. Esta etapa fue realizada con minuciosidad para que las preguntas puedan ser comparables de un año a otro. Esto se llevó a cabo bajo la supervisión y aprobación de la gerencia del área y del proveedor Korn Ferry. La encuesta fue concluida con 61 preguntas para todos los trabajadores, 9 exclusiva para los líderes y 2 preguntas $\operatorname{abiertas}^{2}$.

\subsection{Informar a los trabajadores}

Comunicar a los trabajadores los resultados es una de las etapas más importantes del proceso, ya que, para obtener resultados válidos y confiables, se necesita transparencia y colaboración (Pereda et al., 2014).

Las actividades realizadas en esta etapa del proceso fueron tres: a) se convocó a una reunión de kick off con todos los líderes de la organización para explicar la metodología, etapas y confidencialidad del proceso; b) se solicitó a los líderes, a quienes se les hizo un seguimiento exhaustivo, que comuniquen los resultados de la encuesta a sus equipos, ya que el año anterior no se había logrado del todo; c) vía escenarios técnicos (mailing y WhatsApp), se informó a toda la empresa del proceso y las fechas en que se realizaría la encuesta ${ }^{3}$.

\footnotetext{
2 Ver apéndice 3

${ }^{3}$ Ver apéndice 4
} 


\section{$2.4 \quad$ Recoger los datos}

Para la recogida de datos, es ideal trabajar con todas las personas de la organización; en caso no sea posible, se debe escoger un grupo representativo o el colectivo donde se hayan detectado los problemas (Pereda et al., 2014). Además, en este punto, es fundamental garantizar la confidencialidad para no comprometer el volumen, calidad y sinceridad de respuestas (Bordas, 2016).

Las actividades realizadas por la posición para recoger los datos fueron cinco: a) se definió que todas las personas de la organización participarían de la encuesta; b) se escogió la fecha en que sería la encuesta, que, en caso ideal, hubiera sido en la misma semana que en el año anterior, pero, debido a la Copa Mundial de Fútbol, muchos trabajadores habían solicitado vacaciones para esas fechas, y, además, podrían intervenir variables extrañas debido a la coyuntura del momento, por lo que se solicitó aprobación para realizar la encuesta un mes antes; c) la semana en que se aplicó la encuesta, se conversó con el staff de cada área para reforzar la importancia de su opinión, la confidencialidad del proceso y absolver cualquier duda; y d), se realizó seguimiento respectivo mediante mailing y conversaciones con cada área para obtener la mayor cantidad de respuestas posibles.

\subsection{Analizar los resultados}

Los análisis de las respuestas ofrecen conclusiones que permiten identificar atributos que generan satisfacción o insatisfacción a los trabajadores. Mediante este análisis, se priorizan aspectos de la vida laboral para invertir en forma rentable en la mejora (Bordas, 2016).

Los resultados de la encuesta fueron enviados por la consultora la primera semana de junio en un informe; además, se tuvo acceso a una plataforma que permitió obtener los resultados generales, por dimensión, por factor, por pregunta, por segmento, etc. 
Las actividades realizadas por la posición fueron las siguientes: a) analizar los resultados de cada pregunta, comparando las respuestas del 2017 versus 2018, validando los resultados, realizando cruces entre áreas, tiempo de permanencia, matriz de efectividad, etc., para generar hipótesis de las posibles causas de los resultados; b) se identificó los puntos más fuertes por empresa y por áreas, y, además, las dimensiones de mejora por cada una; y c) se realizó un análisis entre lo recurrente entre la organización y lo que era particular de cada área. Como resultado de este análisis, se priorizó las necesidades inmediatas para analizar en el plan de acción posterior.

\subsection{Entregar los resultados a líderes y comunicar a toda la organización}

Para fomentar la aceptación de la encuesta por parte de los empleados, es importante llevar a cabo un proceso de comunicación claro y transparente (Bordas, 2016). Los resultados de clima laboral fueron expuestos por la consultora a los líderes de la organización la segunda semana de junio, y cada líder recibió un usuario y contraseña para revisar los resultados individuales de sus equipos en la plataforma.

Las actividades realizadas por la posición fueron cuatro: a) concertar una reunión posterior con cada líder para enseñarle el uso de la herramienta, b) validar sus resultados, c) generar hipótesis de las posibles causas, y d) generar planes de acción específicos para cada área, de modo que se prioricen los aspectos de la vida laboral para invertir de forma rentable en la mejora. Este fue un ajuste versus el año 2017, cuando solo se realizó seguimiento para que cada líder ingresara al sistema para obtener su data individual, mas no se generó la reunión personalizada.

La tercera semana de junio se realizó el taller de planes de acción con todos los líderes de la organización, en el que se priorizó los aspectos a trabajar de acuerdo con la necesidad del negocio. Las funciones realizadas por la posición implicaron toda la organización, ejecución y seguimiento a los nuevos planes de acción. Dicho plan de 
acción incluyó acciones que impliquen el involucramiento de los líderes a todo nivel, para que de esta manera se involucren con la cultura y sean agentes de cambio (Gamarra, del Rocío y Paredes Rivera, 2015).

Los resultados fueron comunicados a toda la organización en el mes de julio. Las funciones realizadas por la posición fueron presentar a toda la organización los resultados y el plan de acción a trabajar. 


\section{CAPÍTULO III: RESULTADOS DE LA INTERVENCIÓN}

Los resultados de la intervención ofrecieron un balance positivo. Se cumplió el objetivo general del diagnóstico, pues se gestionó la efectividad organizacional de los trabajadores con el fin de lograr un mejor lugar para trabajar, que contribuya, al mismo tiempo, al logro de los objetivos estratégicos de la empresa. Gracias a la estrategia empleada, se gestionó la efectividad organizacional, y se logró identificar a los trabajadores más efectivos, cuadrante que obtuvo un incremento de 10 puntos versus el año anterior. Se logró que sea un mejor lugar para trabajar, pues se incrementó en 14 puntos el índice de favorabilidad versus el año anterior, con un 83\% de satisfacción. Además, se incrementó 12 puntos sobre el mercado Perú ${ }^{4}$. Entre los objetivos estratégicos del negocio, los ingresos a junio 2018 se incrementaron 4.4\% por encima del 2017; la rentabilidad incrementó en 3\% por encima del 2017; y la publicidad del mall se incrementó en un $29 \%$ por encima del año anterior (Equilibrium Clasificadora de Riesgo, 2018). La gestión positiva del clima organizacional tuvo impacto directamente proporcional con los ingresos y rentabilidad del negocio, lo que se corrobora con investigaciones que concluyen que los buenos resultados empresariales se obtienen por el modo en que se gestionan los recursos humanos (Zenteno-Hidalgo y Durán, 2015).

En cuanto a la actitud de los líderes hacia los resultados de la encuesta, esta fue distinta a la del año anterior, ya que, en esta oportunidad, se consideraron los resultados acertados, válidos y confiables. Este feedback se obtuvo en la reunión de entrega de resultados por áreas, donde cada líder revisó sus resultados de manera personal, brindó retroalimentación del proceso en general y se plantearon planes de acción individuales por áreas de acuerdo con sus necesidades. Los ajustes que marcaron la diferencia en el proceso fueron dos. En primer lugar, la relación con los stakeholders de la encuesta se manejó de manera estratégica, pues los líderes estuvieron involucrados desde la primera etapa, y se consideraron socios estratégicos y no clientes

\footnotetext{
${ }^{4}$ Ver apéndice 5 para la carta de la consultora KF HayGroup
} 
internos; esto generó compromiso, pues se sintieron responsables del éxito del proceso. En segundo lugar, se los involucró en la revisión de la encuesta, como una validación de expertos, lo cual generó mayor compromiso y, a su vez, el hacerlo de manera colaborativa tuvo como consecuencia, incluso, que sean socios para el cumplimiento de sus equipos, pues, a pesar de que la encuesta era voluntaria, se obtuvo una adhesión del 93\%, 4 puntos superior a la del año anterior.

Finalmente, el índice de favorabilidad de la encuesta se encuentra estrechamente relacionado con el rol del líder, dado que investigaciones relacionan al liderazgo como factor principal en la gestión del clima laboral (Bordas, 2016). Ello se vio reflejado en los resultados, ya que las preguntas relacionadas a ese punto tuvieron un incremento porcentual de hasta 28 puntos versus las del año anterior, por ejemplo, en el factor claridad y direccionamiento, en los que los colaboradores indican cómo, desde su trabajo, contribuyen con las metas, objetivos, pilares de la organización y crecimiento del negocio. 


\section{CONCLUSIONES}

- Recursos Humanos es socio del negocio y es responsable de brindar el soporte necesario para que las personas comprometidas puedan ser lo más eficientes posibles $\mathrm{y}$, de esta manera, alcanzar resultados de la organización.

- La gestión positiva del clima organizacional tiene impacto directamente proporcional con los ingresos y rentabilidad del negocio.

- El rol del líder es uno de los principales factores en la gestión del clima laboral.

- Los trabajadores más efectivos son aquellos que cuentan con el soporte de la organización y un grado de compromiso elevado.

- La comunicación fluida durante todo el proceso de diagnóstico de clima laboral, a todo nivel, favorece el éxito de la encuesta.

- Involucrar a los líderes de la organización como socios estratégicos para el desarrollo de la encuesta favorece el cumplimiento y aceptación de la misma. 


\section{RECOMENDACIONES}

- Contar con el apoyo legítimo de la alta dirección al momento de comenzar con el proceso de diagnóstico de clima organizacional

- Involucrar a los líderes de la organización y a todos los stakeholders, desde la primera etapa del proceso de diagnóstico de clima laboral

- Garantizar el anonimato y la confiabilidad del proceso en todas las etapas

- Comunicar oportunamente los resultados del diagnóstico a toda la empresa

- Involucrar a los líderes de la organización en la realización del plan de acción para toda la empresa

- Generar reuniones individuales con los líderes de cada equipo para hacer una entrega de resultados personalizada y hacer, de manera colaborativa, un plan de acción para el área

- Comunicar los planes de acción a todo al personal de manera oportuna

- Cruzar los indicadores directos obtenidos en la encuesta de clima laboral, con los indicadores indirectos como absentismo, rotación, accidentes laborales, productividad, quejas, conflictividad, y aplicarlo a un software de BI.

- Generar planes de acción alineados a lo que la empresa debe reforzar, para generar rentabilidad para el negocio, y, además, que esté alineado a las exigencias del mundo actual, en cuanto a tecnología, ecología y bienestar. 


\section{REFERENCIAS}

América Economía. (5 de abril de 2018). Crecimiento económico de Perú se mantiene alrededor de 3\% del PIB, estima la Cámara de Comercio de Lima. América Economía. Recuperado de https://www.americaeconomia.com/economiamercados/finanzas/crecimiento-economico-de-peru-se-mantiene-alrededor-de-3-delpib-estima

BBC Mundo. (21 marzo de 2018). Perú: renuncia el presidente Pedro Pablo Kuczynski entre acusaciones de corrupción y sobornos. $B B C$. Recuperado de https://www.bbc.com/mundo/noticias-america-latina-43481060

Bordas, M. M. J. (2016). Gestión estratégica del clima laboral. Madrid, España: Universidad Nacional de Educación a Distancia.

CNN Español. (8 marzo de 2017,). ¿En qué va el caso Odebrecht? Cronología interactiva del escándalo. CNN Español. Recuperado de https://cnnespanol.cnn.com/2017/03/07/enque-va-el-caso-odebrecht-cronologia-del-escandalo/

D’Alessio, F. (2017). Pastillas para la Gerencia. Lima, Perú: Planeta.

El Comercio. (2016, 20 septiembre). xxxxxxxxxxxxxxxxxxxxxxxxxxxxxxxxxxxxxxxxxxxx. El Comercio. Recuperado de https://elcomercio.pe/economia/negocios/xxxxxxxxxxxxxxxxxxxxxxxxxxx

Equilibrium Clasificadora de Riesgo. (2018). Informe de Clasificación. Recuperado de 2019 de http://www.equilibrium.com.pe/Xxxxxxxxxxxxxxx 
Gamarra, A., del Rocío, L., y Paredes Rivera, J. M. (2015). Propuesta de mejora de clima organizacional a partir de la gestión del talento humano (Tesis de maestría). Universidad del Pacífico, Lima.

Govea, M., Domínguez, M. M. E., y San Agustín, Y. Q. (2012). Importancia del clima laboral en los resultados de una empresa y la competitividad. Contribuciones a la Economía, 11. Recuperado de http://www.eumed.net/ce/2012/clima-laboral-empresacompetitividad.html

Korn Ferry HayGroup. (2017). Incrementando la Efectividad Organizacional. Propuesta $\operatorname{xxxxxxx} \times x x x x x x x 2017$.

Peña Estrada, C. (2015). La motivación laboral como herramienta de gestión en las organizaciones empresariales. (Tesis de pregrado). Universidad Pontificia Comillas, Madrid.

Pereda, S., Berrocal, F., y Alonso, M. (2014). Bases de Psicología del Trabajo para la gestión de recursos humanos. Madrid: Editorial Síntesis.

RPP Noticias. (28 octubre de 2017). Estas son las cifras oficiales que dejó la emergencia por el Niño Costero a nivel nacional. RPP. Recuperado de https://rpp.pe/politica/gobierno/estas-son-las-cifras-oficiales-que-dejo-la-emergenciapor-el-nino-costero-a-nivel-nacional-noticia-1085350

Robbins, S.P., y Judge, T.A. (2013). Comportamiento organizacional (15ed). México D. F., México: Pearson.

Zenteno-Hidalgo, A., y Durán, C. (2015). Factores y prácticas de alto desempeño que influyen en el clima laboral: análisis de un caso. Innovar: Revista de Ciencias 
Administrativas y Sociales, 26(59), 119-136. Recuperado de https://dx.doi.org/10.15446/innovar.v26n59.54367 
APÉNDICES 


\section{APÉNDICE 1: CARTA DE AUTORIZACIÓN DE LA EMPRESA}

autoriza a SANDRA MARGOTH SIMÓN FERNÁNDEZ, con DNI 45016547 a presentar el Trabajo de suficiencia profesional para optar el título profesional de psicólogo sobre Diagnóstico de Clima Organizacional.

La información presentada es confidencial y su uso es para fines estrictamente académicos.

Atentamente, 


\section{APÉNDICE 2: CRONOGRAMA DE TRABAJO}

\section{Cronograma Encuesta Clima Laboral 2018}

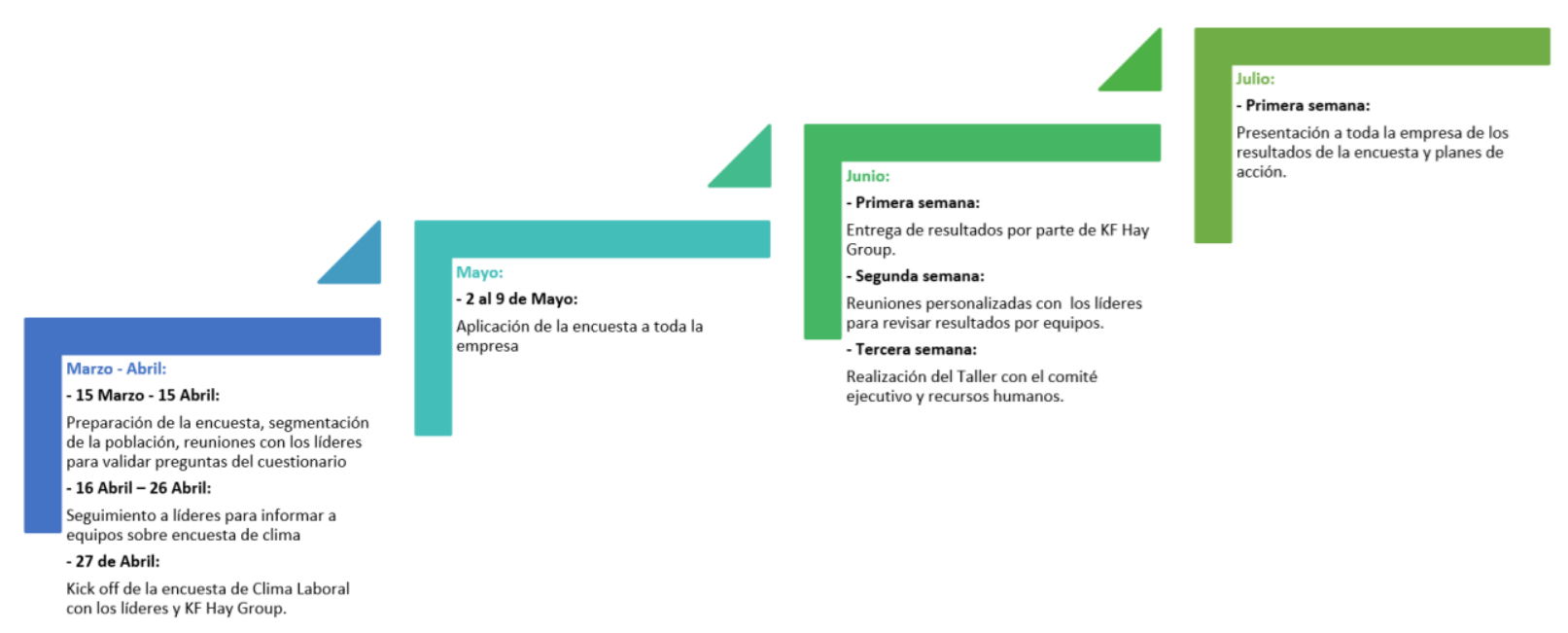




\section{APÉNDICE 3: PREGUNTAS DE LA ENCUESTA}

\begin{tabular}{|c|c|c|c|c|}
\hline & Pregunta & Dimensión & Factor & Versión \\
\hline 1 & $\begin{array}{l}\text { Mi trabajo me permite realizar tareas interesantes y } \\
\text { desafiantes }\end{array}$ & Soporte para el Éxito & $\begin{array}{l}\text { Adecuación al } \\
\text { Cargo }\end{array}$ & Todos \\
\hline 2 & $\begin{array}{l}\text { Mis aptitudes y habilidades son aprovechadas en el cargo } \\
\text { que ocupo }\end{array}$ & Soporte para el Éxito & $\begin{array}{l}\text { Adecuación al } \\
\text { Cargo }\end{array}$ & Todos \\
\hline 3 & $\begin{array}{l}\text { me brinda las condiciones necesarias en mi } \\
\text { trabajo para ser lo más productivo que puedo ser }\end{array}$ & Soporte para el Éxito & $\begin{array}{l}\text { Ambiente } \\
\text { Facilitador } \\
\end{array}$ & Todos \\
\hline 4 & $\begin{array}{l}\text { Puedo realizar mi trabajo efectivamente sin obstáculos que } \\
\text { lo impidan }\end{array}$ & Soporte para el Éxito & $\begin{array}{l}\text { Ambiente } \\
\text { Facilitador } \\
\end{array}$ & Todos \\
\hline 5 & $\begin{array}{l}\text { Tengo la autonomía suficiente para realizar mi trabajo de } \\
\text { manera efectiva }\end{array}$ & $\begin{array}{l}\text { Conductores de } \\
\text { Soporte para el Éxito }\end{array}$ & $\begin{array}{l}\text { Autonomía y } \\
\text { Empowerment }\end{array}$ & Todos \\
\hline 6 & $\begin{array}{l}\text { Existe trabajo en equipo y buena cooperación dentro de } \\
\text { mi área }\end{array}$ & $\begin{array}{l}\text { Conductores de } \\
\text { Soporte para el Éxito }\end{array}$ & Colaboración & Todos \\
\hline 7 & $\begin{array}{l}\text { brinda capacitaciones necesarias para realizar } \\
\text { adecuadamente mi trabajo }\end{array}$ & $\begin{array}{l}\text { Conductores de } \\
\text { Soporte para el Éxito }\end{array}$ & Entrenamiento & Todos \\
\hline 8 & En mi área, el trabajo está bien organizado & $\begin{array}{l}\text { Conductores de } \\
\text { Soporte para el Éxito }\end{array}$ & \begin{tabular}{|l|} 
Estructura y \\
Procesos
\end{tabular} & Todos \\
\hline 9 & $\begin{array}{l}\text { Cuanto mejor sea mi desempeño, } \\
\text { mejor remuneración }\end{array}$ & $\begin{array}{l}\text { Conductores de } \\
\text { Soporte para el Éxito }\end{array}$ & $\begin{array}{l}\text { Gestión del } \\
\text { Desempeño }\end{array}$ & Todos \\
\hline 10 & $\begin{array}{l}\text { En existen oportunidades para alcanzar mis } \\
\text { objetivos profesionales }\end{array}$ & $\begin{array}{l}\text { Conductores de } \\
\text { Compromiso }\end{array}$ & $\begin{array}{l}\text { Oportunidades de } \\
\text { Desarrollo }\end{array}$ & Todos \\
\hline 11 & $\begin{array}{l}\text { Otras áreas, me brindan la información necesaria para } \\
\text { hacer bien mi trabajo }\end{array}$ & $\begin{array}{l}\text { Conductores de } \\
\text { Soporte para el Éxito }\end{array}$ & Recursos & Todos \\
\hline 12 & $\begin{array}{l}\text { Mi equipo, me brinda la información necesaria para hacer } \\
\text { mi trabajo }\end{array}$ & $\begin{array}{l}\text { Conductores de } \\
\text { Soporte para el Éxito }\end{array}$ & Recursos & Todos \\
\hline 13 & $\begin{array}{l}\text { Considerando el puesto que ocupo, mi remuneración es } \\
\text { adecuada en relación a otras empresas }\end{array}$ & $\begin{array}{l}\text { Conductores de } \\
\text { Compromiso }\end{array}$ & $\begin{array}{l}\text { Remuneración y } \\
\text { Beneficios }\end{array}$ & Todos \\
\hline 14 & \begin{tabular}{|l|} 
promueve el equilibrio entre mi vida profesional \\
y personal
\end{tabular} & $\begin{array}{l}\text { Conductores de } \\
\text { Compromiso }\end{array}$ & $\begin{array}{l}\text { Respeto y } \\
\text { Reconocimiento }\end{array}$ & Todos \\
\hline 15 & $\begin{array}{l}\text { Confío en la proyección del negocio para los próximos } 3 \\
\text { años }\end{array}$ & $\begin{array}{l}\text { Conductores de } \\
\text { Compromiso }\end{array}$ & \begin{tabular}{l|} 
Claridad y \\
Direccionamiento
\end{tabular} & Todos \\
\hline 16 & $\begin{array}{l}\text { En términos generales, los gerentes lideran la organización } \\
\text { de manera efectiva }\end{array}$ & $\begin{array}{l}\text { Conductores de } \\
\text { Compromiso }\end{array}$ & $\begin{array}{l}\text { Confianza en los } \\
\text { líderes }\end{array}$ & Todos \\
\hline 17 & $\begin{array}{l}\text { Los nuevos empleados reciben el soporte y entrenamiento } \\
\text { necesario para realizar su trabajo }\end{array}$ & $\begin{array}{l}\text { Conductores de } \\
\text { Soporte para el Éxito }\end{array}$ & Entrenamiento & Todos \\
\hline 18 & \begin{tabular}{|l} 
establece objetivos retadores para los \\
empleados
\end{tabular} & $\begin{array}{l}\text { Conductores de } \\
\text { Soporte para el Éxito }\end{array}$ & $\begin{array}{l}\text { Gestión del } \\
\text { Desempeño }\end{array}$ & Todos \\
\hline 19 & \begin{tabular}{|l|l|} 
En & existen oportunidades de crecimiento \\
profesional como las promociones, rotaciones entre áreas,
\end{tabular} & $\begin{array}{l}\text { Conductores de } \\
\text { Compromiso }\end{array}$ & $\begin{array}{l}\text { Oportunidades de } \\
\text { Desarrollo }\end{array}$ & Todos \\
\hline 20 & $\begin{array}{l}\text { Tengo los recursos y herramientas necesarios para hacer } \\
\text { mi trabajo de manera efectiva }\end{array}$ & $\begin{array}{l}\text { Conductores de } \\
\text { Soporte para el Éxito }\end{array}$ & Recursos & Todos \\
\hline 21 & $\begin{array}{l}\text { Los beneficios otorgados por satisfacen mis } \\
\text { necesidades }\end{array}$ & $\begin{array}{l}\text { Conductores de } \\
\text { Compromiso }\end{array}$ & $\begin{array}{l}\text { Remuneración y } \\
\text { Beneficios }\end{array}$ & Todos \\
\hline 22 & hienect. & $\begin{array}{l}\text { Conductores de } \\
\text { Compromiso }\end{array}$ & $\begin{array}{l}\text { Respeto y } \\
\text { Reconocimiento }\end{array}$ & Todos \\
\hline 23 & $\begin{array}{l}\text { Entiendo cómo mi trabajo contribuye con las metas, } \\
\text { objetivos y pilares estratégicos del }\end{array}$ & $\begin{array}{l}\text { Conductores de } \\
\text { Compromiso }\end{array}$ & \begin{tabular}{|l|} 
Claridad y \\
Direccionamiento
\end{tabular} & Todos \\
\hline 24 & $\begin{array}{l}\text { Mi jefe directo se comunica de forma abierta y honesta } \\
\text { con el equipo }\end{array}$ & $\begin{array}{l}\text { Conductores de } \\
\text { Compromiso }\end{array}$ & $\begin{array}{l}\text { Confianza en los } \\
\text { líderes }\end{array}$ & Todos \\
\hline 25 & $\begin{array}{l}\text { En general, los gerentes de la Empresa se comunican de } \\
\text { forma abierta y honesta con los colaboradores. }\end{array}$ & $\begin{array}{l}\text { Conductores de } \\
\text { Compromiso }\end{array}$ & $\begin{array}{l}\text { Confianza en los } \\
\text { líderes }\end{array}$ & Todos \\
\hline
\end{tabular}




\begin{tabular}{|c|c|c|c|c|}
\hline & Pregunta & Dimensión & Factor & Versión \\
\hline 26 & $\begin{array}{l}\text { Tengo el tiempo necesario para aprovechar oportunidades } \\
\text { de capacitación y desarrollo en }\end{array}$ & $\begin{array}{l}\text { Conductores de } \\
\text { Soporte para el Éxito }\end{array}$ & Entrenamiento & Todos \\
\hline 27 & $\begin{array}{l}\text { Me reúno con mi jefe directo con frecuencia para } \\
\text { conversar sobre cómo realizo mi trabajo }\end{array}$ & $\begin{array}{l}\text { Conductores de } \\
\text { Soporte para el Éxito }\end{array}$ & $\begin{array}{l}\text { Gestión del } \\
\text { Desempeño }\end{array}$ & Todos \\
\hline 28 & $\begin{array}{l}\text { Mi jefe directo dedica tiempo acompañándome en mi } \\
\text { desarrollo profesional }\end{array}$ & $\begin{array}{l}\text { Conductores de } \\
\text { Compromiso }\end{array}$ & $\begin{array}{l}\text { Oportunidades de } \\
\text { Desarrollo }\end{array}$ & Todos \\
\hline 29 & $\begin{array}{l}\text { En relación al trabajo que realizo considero que mi } \\
\text { remuneración es adecuada }\end{array}$ & $\begin{array}{l}\text { Conductores de } \\
\text { Compromiso }\end{array}$ & $\begin{array}{l}\text { Remuneración y } \\
\text { Beneficios }\end{array}$ & Todos \\
\hline 30 & $\begin{array}{l}\text { Recibo reconocimiento de mi jefe directo cuando realizo } \\
\text { un buen trabajo }\end{array}$ & $\begin{array}{l}\text { Conductores de } \\
\text { Compromiso }\end{array}$ & $\begin{array}{l}\text { Respeto y } \\
\text { Reconocimiento }\end{array}$ & Todos \\
\hline 31 & reconoce cuando realizo un buen trabajo & $\begin{array}{l}\text { Conductores de } \\
\text { Compromiso }\end{array}$ & $\begin{array}{l}\text { Respeto y } \\
\text { Reconocimiento }\end{array}$ & Todos \\
\hline 32 & Confío en mi jefe directo & $\begin{array}{l}\text { Conductores de } \\
\text { Compromiso }\end{array}$ & $\begin{array}{l}\text { Confianza en los } \\
\text { líderes }\end{array}$ & Todos \\
\hline 33 & $\begin{array}{l}\text { En general, tengo confianza en los gerentes de esta } \\
\text { Empresa }\end{array}$ & $\begin{array}{l}\text { Conductores de } \\
\text { Compromiso }\end{array}$ & $\begin{array}{l}\text { Confianza en los } \\
\text { líderes }\end{array}$ & Todos \\
\hline 34 & $\begin{array}{l}\text { Tengo una clara idea de los resultados que se esperan de } \\
\text { mi trabajo }\end{array}$ & $\begin{array}{l}\text { Conductores de } \\
\text { Soporte para el Éxito }\end{array}$ & $\begin{array}{l}\text { Gestión del } \\
\text { Desempeño }\end{array}$ & Todos \\
\hline 35 & Soy tratado con respeto & $\begin{array}{l}\text { Conductores de } \\
\text { Compromiso }\end{array}$ & $\begin{array}{l}\text { Respeto y } \\
\text { Reconocimiento }\end{array}$ & Todos \\
\hline 36 & $\begin{array}{l}\text { me motiva a contribuir más allá de lo que mi } \\
\text { cargo requiere }\end{array}$ & Compromiso & $\begin{array}{l}\text { Esfuerzo } \\
\text { Discrecional } \\
\end{array}$ & Todos \\
\hline 37 & Recomendaría omo un buen lugar para trabajar & Compromiso & Compromiso & Todos \\
\hline 38 & Siento orgullo de trabajar en el & Compromiso & Compromiso & Todos \\
\hline 39 & $\begin{array}{l}\text { Me siento motivado por hacer más de lo que mi cargo me } \\
\text { exige }\end{array}$ & Compromiso & $\begin{array}{l}\text { Esfuerzo } \\
\text { Discrecional }\end{array}$ & Todos \\
\hline 40 & $\begin{array}{l}\text { La cantidad de personas que integran mi área es adecuada } \\
\text { en relación al volumen de trabajo existente }\end{array}$ & $\begin{array}{l}\text { Conductores de } \\
\text { Soporte para el Éxito }\end{array}$ & Recursos & Todos \\
\hline 41 & $\begin{array}{l}\text { es innovador en sus métodos de trabajo (uso de } \\
\text { nuevas tecnologías, mejora de procesos internos, entre }\end{array}$ & $\begin{array}{l}\text { Conductores de } \\
\text { Soporte para el Éxito }\end{array}$ & \begin{tabular}{|l|} 
Estructura y \\
Procesos
\end{tabular} & Todos \\
\hline 42 & $\begin{array}{l}\text { Los gerentes de la compañía promueven el intercambio de } \\
\text { ideas y recursos entre las distintas áreas de la organización }\end{array}$ & $\begin{array}{l}\text { Conductores de } \\
\text { Soporte para el Éxito }\end{array}$ & Colaboración & Todos \\
\hline 43 & $\begin{array}{l}\text { Existen oportunidades para que mis ideas sean aceptadas e } \\
\text { implementadas }\end{array}$ & $\begin{array}{l}\text { Conductores de } \\
\text { Soporte para el Éxito }\end{array}$ & $\begin{array}{l}\text { Autonomía y } \\
\text { Empowerment }\end{array}$ & Todos \\
\hline 44 & $\begin{array}{l}\text { me incentiva para proponer nuevas y mejores } \\
\text { ideas para hacer las cosas }\end{array}$ & $\begin{array}{l}\text { Conductores de } \\
\text { Soporte para el Éxito }\end{array}$ & $\begin{array}{l}\text { Autonomía y } \\
\text { Empowerment }\end{array}$ & Todos \\
\hline 45 & $\begin{array}{l}\text { Er los clientes (locatario y proveedor) son } \\
\text { considerados socios y la atención es adecuada en términos }\end{array}$ & $\begin{array}{l}\text { Conductores de } \\
\text { Compromiso }\end{array}$ & $\begin{array}{l}\text { Calidad y } \\
\text { Orientación al }\end{array}$ & Todos \\
\hline 46 & $\begin{array}{l}\text { De acuerdo al momento actual de la Empresa, considero } \\
\text { que la estrategia, los objetivos e iniciativas son las }\end{array}$ & $\begin{array}{l}\text { Conductores de } \\
\text { Compromiso }\end{array}$ & $\begin{array}{l}\text { Claridad y } \\
\text { Direccionamiento }\end{array}$ & Todos \\
\hline 47 & $\begin{array}{l}\text { Conozco el conjunto de principios y valores } \\
\text { organizacionales del Jockey }\end{array}$ & $\begin{array}{l}\text { Conductores de } \\
\text { Compromiso }\end{array}$ & Cultura & Todos \\
\hline 48 & $\begin{array}{l}\text { está organizado y estructurado de manera } \\
\text { efectiva }\end{array}$ & $\begin{array}{l}\text { Conductores de } \\
\text { Soporte para el Éxito }\end{array}$ & \begin{tabular}{|l|} 
Estructura y \\
Procesos
\end{tabular} & Todos \\
\hline 49 & $\begin{array}{l}\text { Mi equipo de trabajo recibe el apoyo adecuado de las } \\
\text { áreas de soporte con las que interactúa }\end{array}$ & $\begin{array}{l}\text { Conductores de } \\
\text { Soporte para el Éxito }\end{array}$ & Colaboración & Todos \\
\hline 50 & \begin{tabular}{|c|} 
está orientado hacia el cliente (consumidor \\
final): comprende y satisface sus necesidades y exigencias
\end{tabular} & $\begin{array}{l}\text { Conductores de } \\
\text { Compromiso }\end{array}$ & $\begin{array}{l}\text { Calidad y } \\
\text { Orientación al }\end{array}$ & Todos \\
\hline
\end{tabular}




\begin{tabular}{|c|c|c|c|c|}
\hline & Pregunta & Dimensión & Factor & Versión \\
\hline 51 & $\begin{array}{l}\text { tiene planes bien definidos para lograr los } \\
\text { objetivos }\end{array}$ & $\begin{array}{l}\text { Conductores de } \\
\text { Compromiso }\end{array}$ & \begin{tabular}{|l|} 
Claridad y \\
Direccionamiento
\end{tabular} & Todos \\
\hline 52 & Mi área está alineada con los objetivos de la organización & $\begin{array}{l}\text { Conductores de } \\
\text { Compromiso }\end{array}$ & $\begin{array}{l}\text { Claridad y } \\
\text { Direccionamiento }\end{array}$ & Todos \\
\hline 53 & \begin{tabular}{|l|} 
Los valores organizacionale \\
son practicados en el día a día
\end{tabular} & $\begin{array}{l}\text { Conductores de } \\
\text { Compromiso }\end{array}$ & Cultura & Todos \\
\hline 54 & $\begin{array}{l}\text { En esta empresa se asignan los recursos (presupuesto, } \\
\text { tiempo, etc.) necesarios para la innovación. }\end{array}$ & \begin{tabular}{l|} 
Conductores de \\
Soporte para el Éxito
\end{tabular} & Innovación & Todos \\
\hline 55 & ofrece servicios y/o productos de alta calidad & $\begin{array}{l}\text { Conductores de } \\
\text { Compromiso }\end{array}$ & $\begin{array}{l}\text { Calidad y } \\
\text { Orientación al }\end{array}$ & Todos \\
\hline 56 & $\begin{array}{l}\text { Los gerentes se comportan en forma consistente con los } \\
\text { valores de la compañía. }\end{array}$ & $\begin{array}{l}\text { Conductores de } \\
\text { Compromiso } \\
\end{array}$ & Cultura & Todos \\
\hline 57 & $\begin{array}{l}\text { Las innovaciones que realiza la empresa en sus servicios } \\
\text { y/o productos son exitosas para el negocio. }\end{array}$ & \begin{tabular}{|l|} 
Conductores de \\
Soporte para el Éxito \\
\end{tabular} & Innovación & Todos \\
\hline 58 & $\begin{array}{l}\text { Las personas de mi equipo están comprometidas a } \\
\text { entregar productos y/o servicios de alta calidad }\end{array}$ & $\begin{array}{l}\text { Conductores de } \\
\text { Compromiso } \\
\end{array}$ & $\begin{array}{l}\text { Calidad y } \\
\text { Orientación al }\end{array}$ & Todos \\
\hline 59 & $\begin{array}{l}\text { Conozco el código de ética y los canales para consultas } \\
\text { y/o denuncias }\end{array}$ & \begin{tabular}{|l|} 
Conductores de \\
Soporte para el Éxito
\end{tabular} & Comunicación & Todos \\
\hline 60 & $\begin{array}{l}\text { Confío en los canales implementados por la empresa para } \\
\text { hacer consultas y/o denuncias con respecto a temas éticos }\end{array}$ & \begin{tabular}{|l|} 
Conductores de \\
Soporte para el Éxito
\end{tabular} & Comunicación & Todos \\
\hline 61 & $\begin{array}{l}\text { La empresa se asegura de que el trabajo que realizo lo } \\
\text { haga bajo condiciones seguras }\end{array}$ & $\begin{array}{l}\text { Conductores de } \\
\text { Soporte para el Éxito }\end{array}$ & $\begin{array}{l}\text { Estructura y } \\
\text { Procesos }\end{array}$ & Todos \\
\hline 62 & $\begin{array}{l}\text { En general, las decisiones en esta empresa se toman sin } \\
\text { retrasos y facilitando el logro de los resultados. }\end{array}$ & $\begin{array}{l}\text { Efectividad } \\
\text { Estratégica. }\end{array}$ & $\begin{array}{l}\text { Excelencia } \\
\text { Operativa }\end{array}$ & Líderes \\
\hline 63 & $\begin{array}{l}\text { Considero que mi empresa es exitosa en el proceso de } \\
\text { captar, desarrollar y mantener a sus clientes (locatario, }\end{array}$ & $\begin{array}{l}\text { Efectividad } \\
\text { Estratégica. }\end{array}$ & $\begin{array}{l}\text { Relación con el } \\
\text { Cliente }\end{array}$ & Líderes \\
\hline 64 & $\begin{array}{l}\text { La empresa destina recursos (financieros y no financieros) } \\
\text { para que los empleados puedan brindar a los clientes }\end{array}$ & $\begin{array}{l}\text { Efectividad } \\
\text { Estratégica. }\end{array}$ & $\begin{array}{l}\text { Relación con el } \\
\text { Cliente }\end{array}$ & Líderes \\
\hline 65 & $\begin{array}{l}\text { Considero que el desempeño financiero de mi empresa } \\
\text { (ingresos, márgenes y utilidades) es mejor o está por }\end{array}$ & $\begin{array}{l}\text { Efectividad } \\
\text { Estratégica. }\end{array}$ & $\begin{array}{l}\text { Excelencia } \\
\text { Operativa }\end{array}$ & Líderes \\
\hline 66 & $\begin{array}{l}\text { La empresa es eficiente para comprender los cambios del } \\
\text { entorno (competidores, mercado, tecnología, etc.) y }\end{array}$ & \begin{tabular}{|l} 
Efectividad \\
Estratégica.
\end{tabular} & $\begin{array}{l}\text { Excelencia } \\
\text { Operativa }\end{array}$ & Líderes \\
\hline 67 & $\begin{array}{l}\text { Los planes en mi empresa son ejecutados dentro de los } \\
\text { plazos y estándares definidos. }\end{array}$ & $\begin{array}{l}\text { Efectividad } \\
\text { Estratégica. }\end{array}$ & $\begin{array}{l}\text { Excelencia } \\
\text { Operativa }\end{array}$ & Líderes \\
\hline 68 & $\begin{array}{l}\text { Los valores de esta compañía son considerados muy } \\
\text { importantes y facilitan el desarrollo del negocio. }\end{array}$ & $\begin{array}{l}\text { Efectividad } \\
\text { Estratégica. }\end{array}$ & Cultura & Líderes \\
\hline 69 & $\begin{array}{l}\text { Las innovaciones que realiza mi empresa cumplen con las } \\
\text { expectativas de ingresos y márgenes financieros }\end{array}$ & $\begin{array}{l}\text { Efectividad } \\
\text { Estratégica. }\end{array}$ & Innovación & Líderes \\
\hline 70 & La empresa es eficiente en el uso de sus recursos. & $\begin{array}{l}\text { Efectividad } \\
\text { Estratégica. }\end{array}$ & $\begin{array}{l}\text { Excelencia } \\
\text { Operativa }\end{array}$ & Líderes \\
\hline 71 & ¿Qué es lo que más te gusta de trabaja & \multicolumn{3}{|c|}{ Pregunta abierta } \\
\hline 72 & $\begin{array}{l}\text { ¿Qué aspectos consideras que se deberían trabajar para } \\
\text { mejorar el clima laboral en el }\end{array}$ & \multicolumn{3}{|c|}{ Pregunta abierta } \\
\hline
\end{tabular}




\section{APÉNDICE 4: MODELO CORREO INVITACIÓN}

Estimado(a),

Tenemos el agrado de invitarlo a participar de la Encuesta de Clima Organizacional del xxxxxxxxxxxx, la cual se desarrollará entre el miércoles 2 y miércoles 9 de Mayo, en conjunto con la Consultora KF HayGroup.

El objetivo de este estudio es conocer cómo es percibida nuestra Compañía por las personas que la integran, sirviendo así como una guía a la gestión futura.

La Consultora KF HayGroup es la responsable de administrar el proceso y garantizar el resguardo y la confidencialidad de toda la información que entregue, lo cual se asegura a través de diversos mecanismos:

- El cuestionario es totalmente anónimo, por lo tanto usted no debe identificarse.

- La información es procesada y analizada por KF HayGroup, quienes clasifican la información en grupos o unidades que tengan un mínimo de 4 personas que contesten la encuesta.

- Se accede al cuestionario directamente desde en el link copiado más abajo. Puede ingresar desde cualquier computador con conexión a Internet, durante las 24 horas del día.

- El link de acceso a la encuesta es único y ha sido generado en forma aleatoria, por lo que no debe compartirla. Una vez que finalice sus respuestas, las claves caducarán automáticamente.

- El sitio web está diseñado para que pueda guardar sus respuestas, con el fin de que pueda completar su cuestionario en otro momento o en su hogar. Al volver a ingresar, será direccionado automáticamente a las preguntas que aún tiene pendientes.

\section{$\underline{\text { Para acceder a la encuesta debe ingresar al siguiente link: }}$}

https://www.haygroupest.com.br/onlinesurvey/Login.rails?login=xxxxxxxx-4590$1101778 \&$ passwd=b58d

Ante cualquier duda sobre la encuesta y el proceso, contáctese directamente con Sandra Simón (anexo xxxx)

En caso de requerir ayuda técnica con la página de la encuesta contáctese directamente al email: clima.organizacional@kornferry.com

Es muy importante que usted participe y responda la encuesta con absoluta franqueza, ya que de su colaboración y honestidad depende el éxito de este estudio y de las acciones que de él se puedan desprender.

Desde ya les agradecemos su participación en este importante proceso.

Les saluda atentamente,

Equipo KF HayGroup 


\section{APÉNDICE 5: CARTA DE KF HAYGROUP}

1 KORN FERRY

Av. Santo Toribio 173, Edificio Torre Real 8, Of. 1202

San Isidro, Lima, Perú

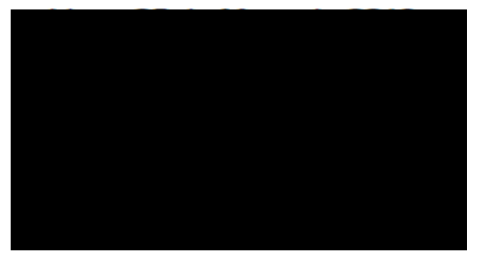

Atención: Srta. Sandra Simon

De nuestra consideración,

Por medio de la presente, le comunicamos que la Encuesta de Clima Laboral del año 2018 correspondiente a la el a consultora ha finalizado con éxito, obteniendo un resultaoo de $83 \%$ de tavoramiloaa globaly un $93 \%$ de adhesión global.

Le enviamos nuestras felicitaciones de parte de todo el equipo de Korn Ferry HayGroup.

Agradeciendo la atención a la presente|

Cordialmente,

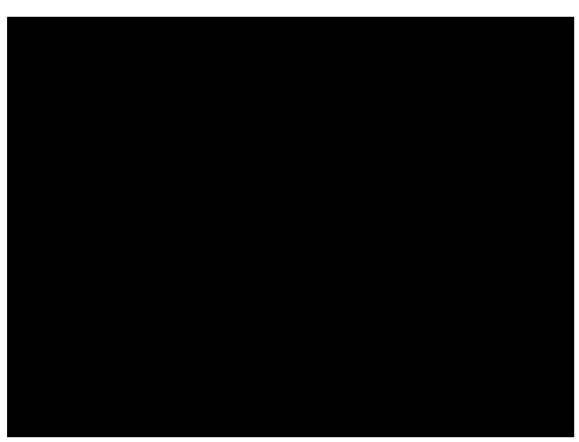

